\title{
Passively Tunable Terahertz Filters Using Liquid Crystal Cells Coated with Metamaterials
}

\author{
Wei-Fan Chiang ${ }^{1}$, Yu-Yun Lu ${ }^{2}$, Yin-Pei Chen ${ }^{2}$, Xin-Yu Lin ${ }^{2}$, Tsong-Shin Lim ${ }^{2}$, Jih-Hsin Liu ${ }^{3}$, Chia-Rong Lee ${ }^{1, * \mathbb{D}}$ \\ and Chia-Yi Huang $2, *(\mathbb{D}$ \\ 1 Department of Photonics, National Cheng Kung University, Tainan 70101, Taiwan; andy03190@gmail.com \\ 2 Department of Applied Physics, Tunghai University, Taichung 40704, Taiwan; s07210048@thu.edu.tw (Y.-Y.L.); \\ s07210044@thu.edu.tw (Y.-P.C.); s07210046@thu.edu.tw (X.-Y.L.); tslim@thu.edu.tw (T.-S.L.) \\ 3 Department of Electrical Engineering, Tunghai University, Taichung 40704, Taiwan; jhliu64@thu.edu.tw \\ * Correspondence: crlee@mail.ncku.edu.tw (C.-R.L.); chiayihuang@thu.edu.tw (C.-Y.H.)
}

Citation: Chiang, W.-F.; Lu, Y.-Y.; Chen, Y.-P.; Lin, X.-Y.; Lim, T.-S.; Liu, J.-H.; Lee, C.-R.; Huang, C.-Y.

Passively Tunable Terahertz Filters Using Liquid Crystal Cells Coated with Metamaterials. Coatings 2021, 11, 381. https://doi.org/10.3390/ coatings11040381

Academic Editor: You Seung Rim

Received: 2 March 2021

Accepted: 25 March 2021

Published: 26 March 2021

Publisher's Note: MDPI stays neutral with regard to jurisdictional claims in published maps and institutional affiliations.

Copyright: (c) 2021 by the authors. Licensee MDPI, Basel, Switzerland. This article is an open access article distributed under the terms and conditions of the Creative Commons Attribution (CC BY) license (https:// creativecommons.org/licenses/by/ $4.0 /)$.

\begin{abstract}
Liquid crystal (LC) cells that are coated with metamaterials are fabricated in this work. The LC directors in the cells are aligned by rubbed polyimide layers, and make angles $\theta$ of $0^{\circ}, 45^{\circ}$, and $90^{\circ}$ with respect to the gaps of the split-ring resonators (SRRs) of the metamaterials. Experimental results display that the resonance frequencies of the metamaterials in these cells increase with an increase in $\theta$, and the cells have a maximum frequency shifting region of $18 \mathrm{GHz}$. Simulated results reveal that the increase in the resonance frequencies arises from the birefringence of the LC, and the LC has a birefringence of 0.15 in the terahertz region. The resonance frequencies of the metamaterials are shifted by the rubbing directions of the polyimide layers, so the LC cells coated with the metamaterials are passively tunable terahertz filters. The passively tunable terahertz filters exhibit promising applications on terahertz communication, terahertz sensing, and terahertz imaging.
\end{abstract}

Keywords: liquid crystals; metamaterials; terahertz filters

\section{Introduction}

Metamaterials have attracted much attention due to their unusual electromagnetic resonance [1-7]. The resonance frequencies of metamaterials are sensitive to their geometrical structures [1-3] and the refractive indices of the media that surround the metamaterials [4-7]. Therefore, metamaterials can be used to develop frequency filters, intensity modulators, and sensors. Liquid crystals (LCs) have been used to tune the resonance frequencies of terahertz metamaterials due to their large birefringences [6,7]. Chen et al. deposited a dual frequency LC layer on a terahertz metamaterial [6]. The resonance spectrum of the terahertz metamaterial can be red-shifted and blue-shifted by switching the frequency of a given voltage because the dual frequency LC exhibits positive and negative dielectric anisotropies at low and high frequencies. Therefore, the dual frequency LC layer that is deposited on the terahertz metamaterial can be used to develop electrically controllable terahertz filters. Deng et al. deposited an LC layer between a terahertz metamaterial and a metal layer [7]. The resonance frequency of the terahertz metamaterial is red-shifted by 11.4 (12.6) $\mathrm{GHz}$ at the irradiation of terahertz waves with TE (TM) polarization as a voltage that is applied to the LC layer increases from zero to $10 \mathrm{~V}$. Therefore, the metamaterial-imbedded LC cell can be used to develop electrically controllable terahertz absorbers.

The application of the large voltages to the LC layers causes the shift of the resonance spectra of the metamaterials in the previous works [6,7]. The electrically controllable terahertz devices in these works consume a lot of power to filter incident terahertz waves. This drawback hinders the usage of the terahertz devices in practical applications. It is of great interest to researchers to develop LC-based metamaterials with zero power consumption. 
This work fabricates LC cells coated with terahertz metamaterials, and studies the effect of the rubbing directions of the alignment layers of the cells on the electromagnetic resonance of the metamaterials. The resonance frequencies of the terahertz metamaterials increase with an increase in the angles between the directors of the LC and the gaps of the SRRs. A finite-difference time-domain simulation depicts that the increase in the resonance frequencies arises from the birefringence of the LC, and the LC has a birefringence of 0.15 in the terahertz region. The LC cells coated with the terahertz metamaterials are passively tunable filters with zero power consumption, and can be used in terahertz imaging, terahertz sensing and terahertz communication.

\section{Materials and Methods}

Figure 1a presents the schematic configuration of LC cells that are coated with terahertz metamaterials. Each of the LC cells are fabricated using two 188- $\mu$ m-thick polyester (PET) substrates, which are separated by two plastic spacers with a thickness of $500 \mu \mathrm{m}$. A 200-nm-thick silver SRR array is coated on one of the PET substrates by photolithography, metal evaporation, and lift-off process. Figure $1 \mathrm{~b}$ presents the dimensions of one of the SRRs in the array. The SRR has a linewidth $(w)$, split gap $(g)$, short side $(s)$, long side $(l)$, period in the $x$ direction, and period in the $y$ direction of $6 \mu \mathrm{m}, 20 \mu \mathrm{m}, 50 \mu \mathrm{m}, 60 \mu \mathrm{m}, 70 \mu \mathrm{m}$, and $80 \mu \mathrm{m}$, respectively. Six-hundred-nanometer-thick polyimide (PI) layers (AL-1426CA, Daily Polymer) are coated on both PET substrates as homogenously aligning layers and are rubbed using a rubbing machine. The directors in the LC cells that are coated with the terahertz metamaterials make angles $\theta$ of $0^{\circ}, 45^{\circ}$, and $90^{\circ}$ with respect to the gaps of the SRRs of the metamaterials. A nematic LC (HTW114200-100, Fusol Material) is used in the cells. The nematic LC has been used to develop high-performance optical devices $[8,9]$. However, the LC has not been used to develop terahertz devices. Therefore, the LC is used in this work. The LC layers with a thickness of $500 \mu \mathrm{m}$ are so thick that terahertz waves experience their refractive indices significantly [10,11]. The LC has ordinary and extraordinary refractive indices of 1.513 and 1.779 , respectively, in the visible light regime.

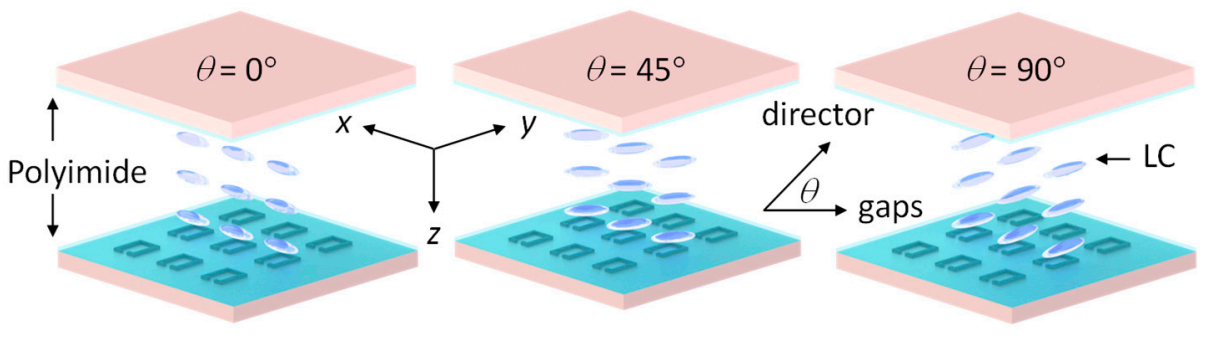

(a)

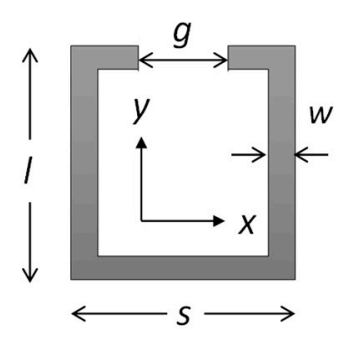

(b)

Figure 1. (a) Schematic configuration of liquid crystal (LC) cells that are coated with terahertz metamaterials. $\theta$ is an angle between the director of the LC and the gaps of the split-ring resonators (SRRs). (b) Dimensions of SRR.

\section{Results and Discussion}

Figure 2 displays the experimental terahertz spectra of three metamaterials that are coated with the PI layers. These spectra are obtained using a commercial terahertz spectrometer (TPS 3000, TeraView, Cambridge, UK) in transmission mode, and the chamber in the spectrometer is filled with nitrogen gas to prevent terahertz waves from absorbing moisture. The polarization of incident terahertz waves is set parallel to the $x$ axis of Figure 1a. The PI-coated metamaterials have transmission peaks in their terahertz spectra due to the absorption of the electromagnetic resonance of the metamaterials. The transmission peaks are at an identical frequency of $0.588 \mathrm{THz}$, and the two PI-coated metamaterials have similar resonance spectra. Therefore, the three PI-coated metamaterials can be used to study the effect of the rubbing directions of the PI layers on the electromagnetic resonance of the metamaterials. 


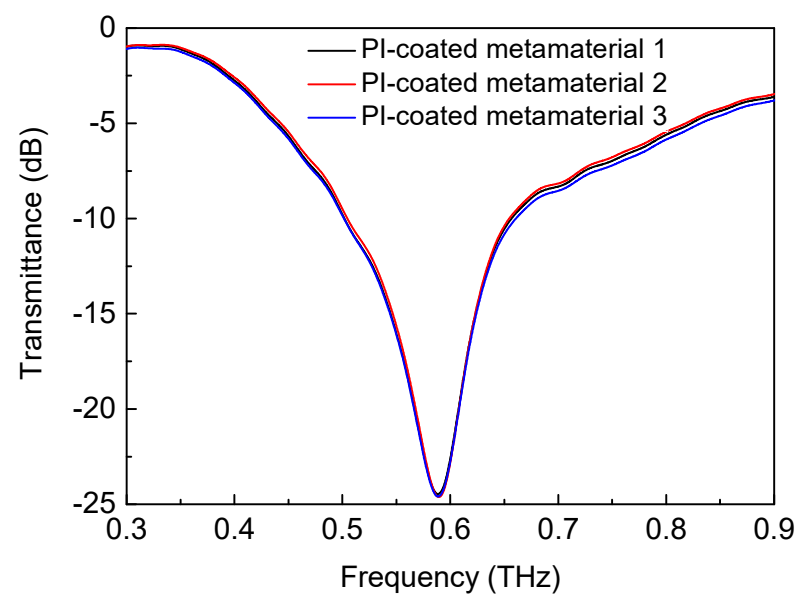

Figure 2. Experimental spectra of three metamaterials that are coated with polyimide (PI) layers.

A simulation is performed using a software based on finite-difference time-domain method to verify the experimental spectra of the PI-coated metamaterials. A simulated SRR has the same geometrical dimensions as the SRR of Figure $1 b$, and is deposited on a $188-\mu \mathrm{m}$-thick PET substrate with an area of $70 \mu \mathrm{m} \times 80 \mu \mathrm{m}$. A 600-nm-thick PI film is coated on the simulated SRR. The permittivity of the PET substrate (PI film) is 3.0 (1.21) in the simulation, and was obtained from its time-domain spectrum. A periodical boundary condition is set in the simulation, and the conductivity of silver in the simulated SRR is $6.30 \times 10^{7} \mathrm{~S} / \mathrm{m}$. Figure 3 displays the simulated spectrum of the PI-coated metamaterials. These metamaterials have a simulated peak at $0.588 \mathrm{THz}$. Therefore, the peak frequency of the simulated spectrum of Figure 3 verifies that of the experimental spectra of Figure 2. The inset in Figure 3 presents the near-field distribution of the simulated SRR that are coated with the PI film at $0.588 \mathrm{THz}$. The near field of this SRR exhibits the maximum strength at its gap. This result depicts that the electromagnetic resonance of the PI-coated metamaterials is an inductive-capacitive mode at $0.588 \mathrm{THz}$.

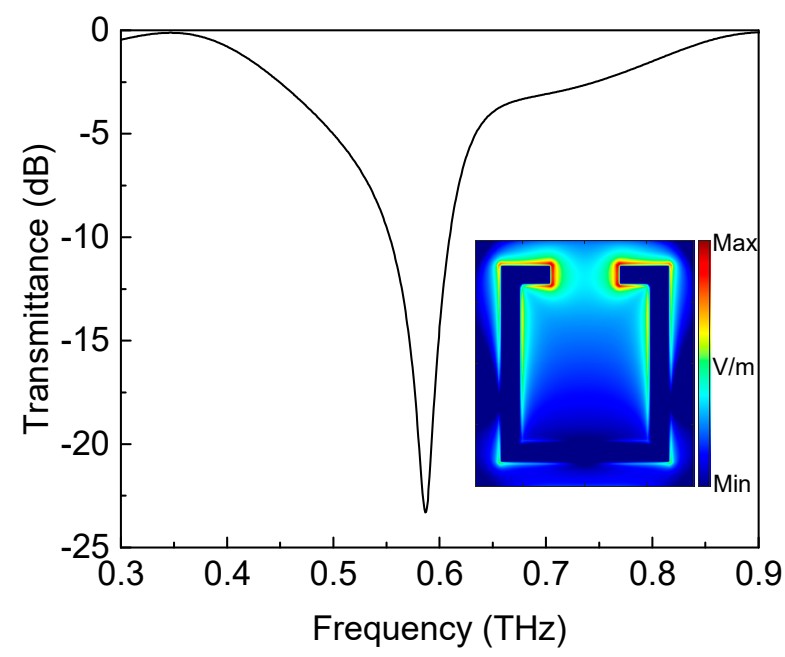

Figure 3. Simulated spectrum of PI-coated metamaterials. The insert presents the near-field distribution of the simulated SRR with the PI film at $0.588 \mathrm{THz}$.

An SRR can be considered as an inductor-capacitor circuit, and its resonance frequency is given by [12]

$$
f=\frac{1}{2 \pi \sqrt{L C}}
$$


where $L$ is an inductance of the inductor, and $C$ is a capacitance of the capacitor. $L$ is determined by the enclosed area of the SRR, and $C$ is proportional to the permittivities of the media that surround the SRR. Equation (1) depicts that the resonance frequency of the inductive-capacitive mode of a metamaterial is inversely proportional to the refractive index of a dielectric layer that is deposited on the metamaterial [12]. Therefore, the PIcoated metamaterials will be sensitive to change in the refractive indices of the dielectric layers that are deposited on them.

Figure 4 displays the experimental spectra of the three LC cells that are coated with the terahertz metamaterials at $\theta=0^{\circ}, 45^{\circ}$, and $90^{\circ}$. The metamaterial-coated LC cells at $\theta=0^{\circ}$, $45^{\circ}$, and $\theta=90^{\circ}$ have resonance peaks at $0.533 \mathrm{THz}, 0.542 \mathrm{THZ}$, and $0.551 \mathrm{THz}$, respectively. The resonance frequencies of the LC cells increase with an increase in $\theta$, and the cells have a maximum frequency-shifting region of $18 \mathrm{GHz}$. The increase in the resonance frequencies arises from the birefringence of the LC. As the cell is at $\theta=0^{\circ}\left(90^{\circ}\right)$, the LC director is parallel (perpendicular) to the gaps of the SRRs of the metamaterial. In addition, the polarized direction of incident terahertz waves is parallel to these gaps. Therefore, the incident terahertz waves experience an extraordinary (ordinary) refractive index of the LC in the cell at $\theta=0^{\circ}\left(90^{\circ}\right)$. This result depicts that the LC has an extraordinary (ordinary) refractive index as the metamaterial has a resonance frequency at $0.533 \mathrm{THz}(0.551 \mathrm{THz})$. Therefore, the LC birefringence causes the increase in the resonance frequencies as the angles between the LC directors and the SRR gaps are increased. Resonance frequencies of plasmonic materials can be tuned by active methods such as external voltage, pump light, and heat or by passive methods such as the change in their geometrical dimensions $[13,14]$. The results in Figure 4 reveal that the resonance frequency of a metamaterial-coated LC cell can be passively tuned by the rubbing direction of its alignment layers. The metamaterialcoated LC cells are passively tunable terahertz filters, and exhibit promising applications on terahertz communication, terahertz sensing, and terahertz imaging. A situation in which photoalignment layers replace the PI layers in this work may fabricate light-controllable terahertz metamaterials. Efforts are being made at the authors' laboratory to fabricate light-controllable terahertz metamaterials, the results of which will be published in the near future.

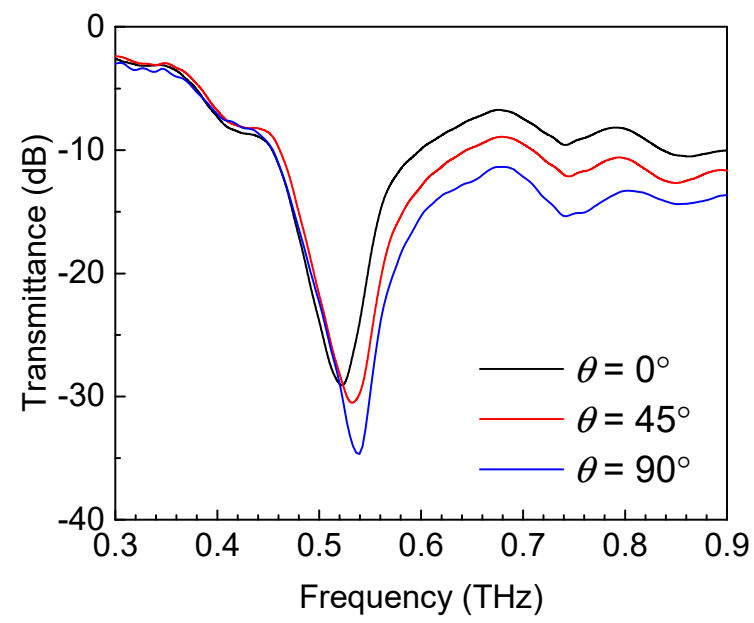

Figure 4. Experimental spectra of three metamaterial-coated LC cells at $\theta=0^{\circ}, 45^{\circ}$, and $90^{\circ}$.

A simulation is performed using software to verify the experimental spectra of the three metamaterial-coated LC cells. A dielectric layer with a refractive index of $n$ is deposited on the simulated PI-coated SRR (the inset of Figure 3). Figure 5 displays the terahertz spectra of the simulated PI-coated SRR with the dielectric layer at $n=1.55,1.62$, and 1.70. This SRR has simulated resonance frequencies of $0.551,0.542$, and $0.533 \mathrm{THz}$ at $n=1.55,1.62$, and 1.70, respectively. The resonance frequency of the simulated PI-coated SRR with the dielectric layer at $n=1.70$ (1.55) equals that of the metamaterial-coated LC 
cell at $\theta=0^{\circ}\left(90^{\circ}\right)$. The polarized direction of the incident terahertz waves is parallel (perpendicular) to the LC director of the metamaterial-coated LC cell at $\theta=0^{\circ}\left(90^{\circ}\right)$, so the LC has an extraordinary (ordinary) refractive index $n_{e}\left(n_{0}\right)$ of $1.70(1.55)$ in the terahertz region. This result reveals that the LC exhibits a birefringence of 0.15 in the terahertz region, and the LC birefringence increases the resonance frequencies of the metamaterials as the angles between the directors and the gaps of their SRRs are increased.

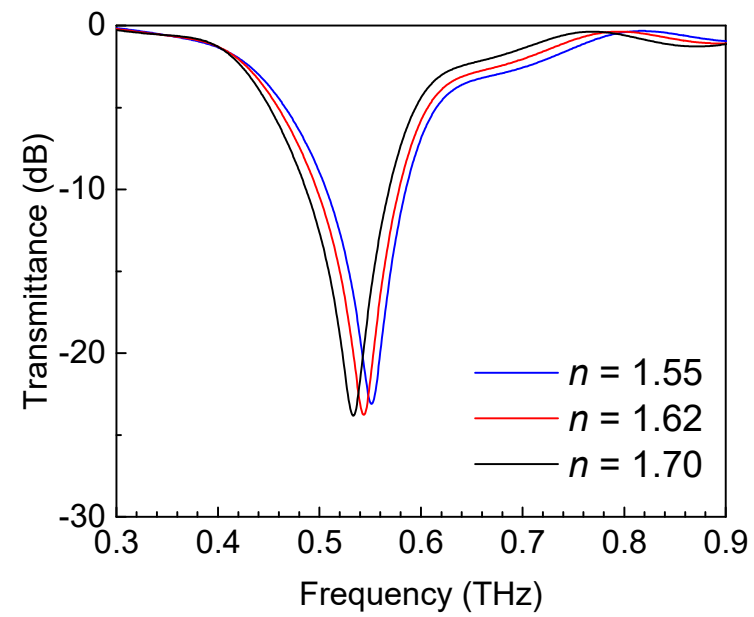

Figure 5. Simulated spectra of three metamaterial-coated LC cells at $n=1.55,1.62$, and 1.70. $n$ is the refractive index of a dielectric layer that is deposited on the simulated PI-coated SRR.

The effective refractive index of the LC in the metamaterial-coated LC cell that involves $\theta=45^{\circ}$ can be determined by an equation of the index ellipse of the LC, and it is given by

$$
n_{e f f}=\sqrt{\frac{1}{\cos ^{2} \theta / n_{e}^{2}+\sin \theta^{2} / n_{o}^{2}}},
$$

where $\theta$ is an angle between the LC director between the polarized direction of the incident terahertz waves. Substituting $n_{e}=1.70, n_{o}=1.55$, and $\theta=45^{\circ}$ into Equation (2) yields $n_{\text {eff }}=1.62 . n_{\text {eff }}$ equals the refractive index of the LC in the metamaterial-coated LC cell at $\theta=45^{\circ}$, as presented in Figure 5. This result verifies that the rubbing directions of the PI layers of the cells can be used to passively tune the resonance frequencies of the metamaterials in the metamaterial-coated LC cells at $\theta=0^{\circ}, 45^{\circ}$, and $90^{\circ}$. The thickness $(600 \mathrm{~nm})$ of the PI layers exceeds that $(200 \mathrm{~nm})$ of the metamaterials. Therefore, the LC layers are anisotropic on the PI layers. This fact reveals that the effective refractive index of the LC in the metamaterial-coated LC cell that involves $\theta=45^{\circ}$ can be calculated from Equation (2).

Figure 4 displays that the transmittances of the metamaterials at their experimental resonance frequencies decrease with an increase in $\theta$. The decrease in the transmittances arises from the anisotropic absorption of the LC in the terahertz region. The terahertz waves are extraordinary (ordinary) waves in the metamaterial-coated LC cell at $\theta=0^{\circ}\left(90^{\circ}\right)$. In addition, most LCs in the terahertz region have a small (large) absorption coefficient for extraordinary (ordinary) waves $[15,16]$. Therefore, the anisotropic absorption of the LC in the terahertz region decreases the experimental resonance transmittances of the metamaterials with the increase in $\theta$. Figure 5 presents that the metamaterial-coated LC cells have similar transmittances at the simulated resonance frequencies of the metamaterials. The similar resonance transmittances arise from the fact that the anisotropic absorption of the LC is not considered in the simulation.

Another LC (E7, Merck, Kenilworth, NJ, USA) is used to study the effect of the birefringence of the LC on the electromagnetic resonance of metamaterials. Metamaterialcoated LC cells have the same geometric configuration as the cells of Figure 1a, but are filled 
with the E7 LC. Figure 6 presents the experimental spectra of the metamaterial-coated cells filled with the E7 LC at $\theta=0^{\circ}$ and $90^{\circ}$. These cells have resonance frequencies of $0.533 \mathrm{THz}$ and $0.542 \mathrm{THz}$ at $\theta=0^{\circ}$ and $\theta=90^{\circ}$, respectively. Therefore, the metamaterial-coated cells filled with the E7 LC have a frequency shifting region of $9 \mathrm{GHz}$.

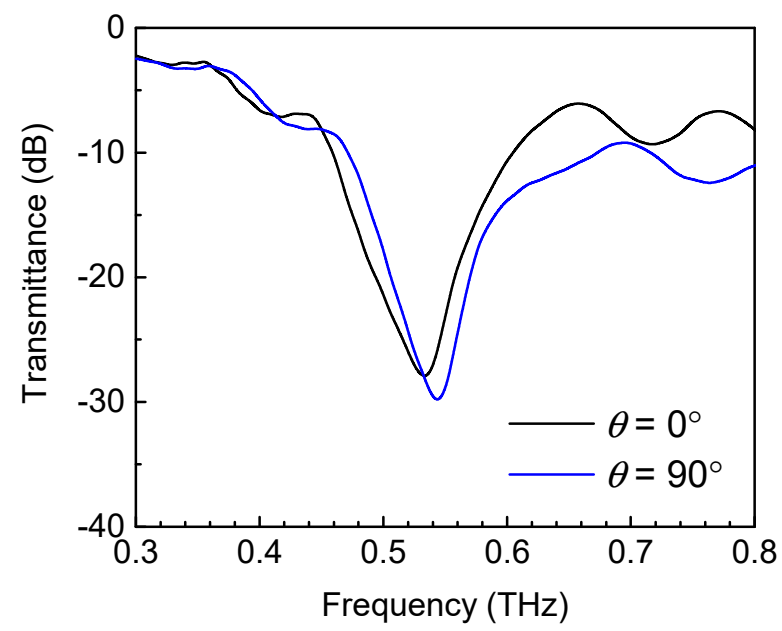

Figure 6. Experimental spectra of metamaterial-coated LC cells filled with E7 LC at $\theta=0^{\circ}$ and $90^{\circ}$.

A simulation is performed using the software to verify the experimental spectra of the metamaterial-coated cells filled with the E7 LC. A dielectric layer with a refractive index of $n$ is deposited on the simulated PI-coated SRR (the inset of Figure 3). Figure 7 displays the terahertz spectra of the simulated PI-coated SRR with the dielectric layer at $n=1.62$ and 1.70. This SRR has simulated resonance frequencies of $0.542 \mathrm{THz}$ and $0.533 \mathrm{THz}$ at $n=1.62$ and 1.70, respectively. The resonance frequency of the simulated PI-coated SRR with the dielectric layer at $n=1.70$ (1.62) equals that of the metamaterial-coated LC cell filled with the E7 LC at $\theta=0^{\circ}\left(90^{\circ}\right)$. In addition, the polarized direction of the incident terahertz waves is parallel (perpendicular) to the LC director in the metamaterial-coated LC cell filled with the E7 LC at $\theta=0^{\circ}\left(90^{\circ}\right)$. Therefore, the E7 LC has an extraordinary (ordinary) refractive index $n_{e}\left(n_{o}\right)$ of 1.70 (1.62) in the terahertz region. This result reveals that the E7 LC exhibits a birefringence of 0.08 in the terahertz region. Because the birefringence (0.08) of the E7 LC is smaller than that (0.15) of the HTW114200-100 LC, the frequency shifting region $(9 \mathrm{GHz})$ of the metamaterial-coated LC cell filled with the former is smaller than that $(18 \mathrm{GHz})$ of the metamaterial-coated LC cell filled with the latter.

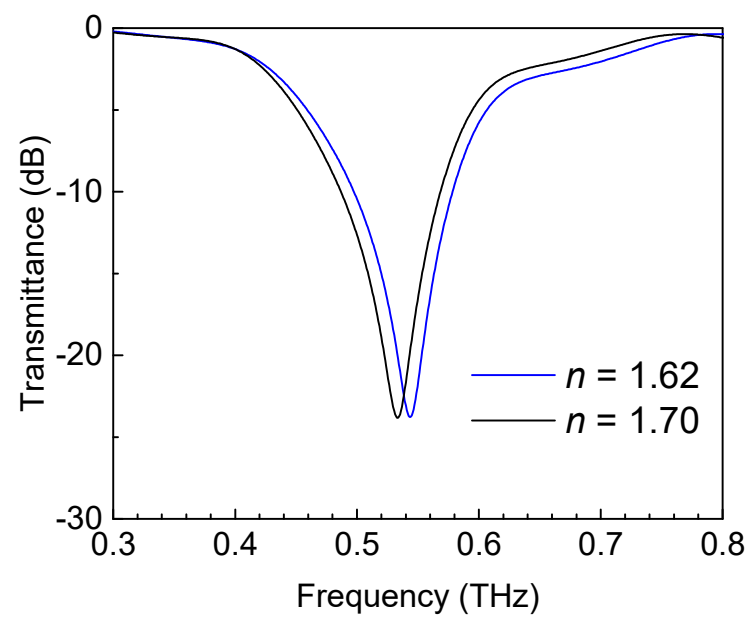

Figure 7. Simulated terahertz spectra of metamaterial-coated LC cells filled with E7 LC at $n=1.62$ and 1.70 . 


\section{Conclusions}

This work fabricated the metamaterial-coated LC cells with the LC directors that make the angles of $0^{\circ}, 45^{\circ}$, and $90^{\circ}$ with respect to the gaps of the SRRs of the metamaterials. They are used to study the effect of the rubbing directions of the PI layers of the cells on the electromagnetic resonance of the metamaterials. The experimental results reveal that the resonance frequencies of the metamaterial-coated LC cells increase with the increase in the angles, and the cells have a maximum frequency shifting region of $18 \mathrm{GHz}$. The simulated results depict that the shift of the resonance spectra of the cells arises from the LC birefringence, and the LC has a birefringence of 0.15 in the terahertz region. The metamaterial-coated LC cells are passively tunable terahertz filters due to the rubbing directions of the PI layers. The passively tunable terahertz filters can be used in terahertz sensing, terahertz communication, and terahertz imaging.

Author Contributions: Conceptualization, C.-Y.H.; methodology, W.-F.C.; software, W.-F.C.; validation, W.-F.C.; formal analysis, W.-F.C., Y.-Y.L., Y.-P.C., X.-Y.L., and C.-Y.H.; investigation, W.-F.C., C.-R.L., and C.-Y.H.; resources, T.-S.L., J.-H.L., and C.-Y.H.; data curation, W.-F.C. and C.-Y.H.; writing-original draft preparation, W.-F.C. and C.-Y.H.; writing-review and editing, W.-F.C. and C.-Y.H.; visualization, W.-F.C. and C.-Y.H.; supervision, C.-R.L. and C.-Y.H.; project administration, C.-Y.H.; funding acquisition, C.-Y.H. All authors have read and agreed to the published version of the manuscript.

Funding: This research was funded by the Ministry of Science and Technology (MOST) of Taiwan under Contract No. MOST 107-2112-M-029-005-MY3.

Institutional Review Board Statement: Not applicable.

Informed Consent Statement: Not applicable.

Data Availability Statement: Data are contained within the article.

Conflicts of Interest: The authors declare no conflict of interest.

\section{References}

1. Xu, T.; Lin, Y.S. Tunable terahertz metamaterial using an electric split-ring resonator with polarization-sensitive characteristic. Appl. Sci. 2020, 10, 4660. [CrossRef]

2. Liao, Y.; Lin, Y.S. Reconfigurable terahertz metamaterial using split-ring meta-atoms with multifunctional electromagnetic characteristics. Appl. Sci. 2020, 10, 5267. [CrossRef]

3. Chen, X.; Lin, Y.S. Polarization-sensitive metamaterials with tunable multi-resonance in the terahertz frequency range. Crystals 2020, 10, 611. [CrossRef]

4. Zhang, Y.; Lin, P.; Lin, Y.S. Tunable Split-Disk Metamaterial Absorber for Sensing Application. Nanomaterials 2021, 11, 598. [CrossRef] [PubMed]

5. Xu, R.; Lin, Y.S. Tunable infrared metamaterial emitter for gas sensing application. Nanomaterials 2020, 10, 1442. [CrossRef] [PubMed]

6. Chen, C.C.; Chiang, W.F.; Tsai, M.C.; Jiang, S.A.; Chang, T.H.; Wang, S.H.; Huang, C.Y. Continuously tunable and fast-response terahertz metamaterials using in-plane-switching dual-frequency liquid crystal cells. Opt. Lett. 2015, 40, 2021-2024. [CrossRef] [PubMed]

7. Deng, G.; Lu, Y.; Yin, Z.; Lai, W.; Lu, H.; Yang, J.; Yang, A.; Ye, Y.; Liu, D.; Chi, B. A Tunable Polarization-Dependent Terahertz Metamaterial Absorber Based on Liquid Crystal. Electronics 2018, 7, 27. [CrossRef]

8. Lin, S.H.; Huang, L.S.; Lin, C.H.; Kuo, C.T. Polarization-independent and fast tunable microlens array based on blue phase liquid crystals. Opt. Express 2014, 22, 925-930. [CrossRef] [PubMed]

9. Jiang, S.A.; Sun, W.J.; Lin, S.H.; Lin, J.D.; Huang, C.Y. Optical and electro-optic properties of polymer-stabilized blue phase liquid crystal cells with photoalignment layers. Opt. Express 2017, 25, 28179-28191. [CrossRef]

10. Pan, C.; Hsieh, C.; Pan, R.; Tanaka, M.; Miyamaru, F.; Tani, M.; Hangyo, M. Control of enhanced THz transmission through metallic hole arrays using nematic liquid crystal. Opt. Express 2005, 13, 3921-3930. [CrossRef] [PubMed]

11. Withayachumnankul, W.; Fischer, B.M.; Abbott, D. Material thickness optimization for transmission-mode terahertz time-domain spectroscopy. Opt. Express 2008, 16, 7382-7396. [CrossRef] [PubMed]

12. O'Hara, J.F.; Singh, R.; Brener, I.; Smirnova, E.; Han, J.; Taylor, A.J.; Zhang, W. Thin-film sensing with planar terahertz metamaterials: Sensitivity and limitations. Opt. Express 2008, 16, 1786-1795. [CrossRef] [PubMed]

13. Wang, B.; Zhai, X.; Wang, G.; Huang, W.; Wang, L. Frequency tunable metamaterial absorber at deep-subwavelength scale. Opt. Mater. Express 2015, 5, 227-235. [CrossRef] 
14. Cao, T.; Wei, C.; Li, Y. Dual-band strong extrinsic 2D chirality in a highly symmetric metal-dielectric-metal achiral metasurface. Opt. Mater. Express 2016, 6, 303-311. [CrossRef]

15. Park, H.; Parrott, E.P.J.; Fan, F.; Lim, M.; Han, H.; Chigrinov, V.G.; Pickwell-MacPherson, E. Evaluating liquid crystal properties for use in terahertz devices. Opt. Express 2012, 20, 11899-11905. [CrossRef] [PubMed]

16. Li, X.; Tan, N.; Pivnenko, M.; Sibik, J.; Zeitler, J.A.; Chu, D. High-birefringence nematic liquid crystal for broadband THz applications. Liq. Cryst. 2016, 43, 955-962. [CrossRef] 